\title{
Palliative Care Involvement in Patients with Operative Mortality After Cardiac Surgery
}

\author{
Hayden S. Leeds, BA, ${ }^{1}$ Danielle A. Smith, $\mathrm{MD}^{2}$ \\ ${ }^{1}$ Division of Cardiothoracic Surgery, Department of Surgery, Oregon Health and Science University, Portland, OR, USA; \\ ${ }^{2}$ Cardiac Surgery Section, Operative Care Division, Portland Veterans Affairs Medical Center, Portland, OR, USA
}

\section{ABSTRACT}

Background: The aim of this study was to examine palliative care involvement in patients with operative mortality after cardiac surgery, to gain a better understanding of this high-risk population and factors influencing referral.

Methods: This is a retrospective review using data submitted to the Society of Thoracic Surgeons National Database.

Results: The total study population included 93 patients with operative mortality, only $30 \%$ of whom had palliative care involvement. The median time from first palliative care involvement to death was 6 days. Nearly $40 \%$ of patients had a status labeled as elective at the time of surgery.

Conclusion: Our findings suggest that patient mortality risk for elective surgery is being underestimated, or the indications for palliative care consultation in high-risk patients is going unrecognized. Such knowledge may facilitate earlier palliative care involvement, with the goal of improved quality of life for cardiac patients.

\section{INTRODUCTION}

The prevalence and impact of cardiovascular disease are at an all-time high, as the population ages and the incidence of comorbid health conditions are on the rise. With advancements in modern medicine, patients with such conditions are living longer and thus being referred for surgical intervention at higher rates [Diop 2017; Warraich 2016; Warraich 2017. Despite such progress, patients with cardiovascular disease continue to experience disproportionate suffering at the end of life [Warraich 2016].

Clinical risk assessment continues to be a challenge for cardiovascular care providers, especially regarding cardiac patients being considered for surgery. Although risk calculators do exist to aid clinicians in quantifying prognosis, it is often near impossible to account for every factor that contributes to an individual's risk, as the prevalence of comorbid conditions continues to increase. Such challenges affect the ability of patients and their families to make important

Received August 9, 2020; accepted September 1, 2020.

Correspondence: Hayden S. Leeds, Division of Cardiothoracic Surgery, Department of Surgery, Oregon Health and Science University, Portland, OR, USA (e-mail: leedsh@obsu.edu). decisions regarding care and involve palliative care [Warraich 2016; Warraich 2017].

Professional societies, such as the American College of Surgeons, have issued calls to action regarding palliative care involvement in the care of high-risk surgical patients [Lilley 2018]. Despite this strong recommendation, palliative care services remain underutilized in surgical and cardiovascular patients [Warraich 2019]. When incorporated, such services are often involved late in the illness trajectory, despite evidence showing their utility in these patient populations. A recent study found that one-third of patients with cardiovascular disease who were referred for palliative care were bedbound at the time of referral [Warraich 2019].

Surgical patients have even lower rates of palliative care involvement [Lilley 2018], and few studies have explored palliative care involvement in patients undergoing cardiac surgery. Thus, the aim of this study was to examine palliative care involvement in patients who died after open-heart surgery at an academic institution, to gain a better understanding of this high-risk population and factors influencing referral. Our hope is that such insight will help guide future preoperative counseling and institutional practices.

Table 1. Demographics*

\begin{tabular}{lc}
\hline Mean age in years (range) & $64(24$ to 88$)$ \\
Male sex & 67.7 \\
White race & 89.5 \\
Mean body surface area $\left(\mathrm{m}^{2}\right)$ & 2.0 \\
Comorbid conditions & \\
Hypertension & 44.8 \\
Diabetes & 36.5 \\
Dyslipidemia & 35.4 \\
Chronic lung disease & 15.6 \\
Cerebrovascular disease & 14.6 \\
Peripheral vascular disease & 13.5 \\
Cancer within 5 years & 5.2 \\
ESRD requiring dialysis & 2.0
\end{tabular}

*Data are \% unless noted otherwise. ESRD indicates end-stage renal disease. 
Table 2. Characteristics of Study Participants*

\begin{tabular}{|c|c|c|}
\hline & All & $\begin{array}{l}\text { Palliative Care } \\
\text { Consultation }\end{array}$ \\
\hline \multicolumn{3}{|l|}{ Palliative care consultation } \\
\hline Total & $28(30.1)$ & \\
\hline Preoperative & $3(3.2)$ & \\
\hline Postoperative & $20(21.5)$ & \\
\hline Both & $5(5.4)$ & \\
\hline \multicolumn{3}{|l|}{ Surgery type $(n>1)$} \\
\hline Aortic dissection & $18(19.3)$ & $4(22.2)$ \\
\hline CABG & $15(16.1)$ & $5(33.3)$ \\
\hline AVR & $10(10.8)$ & $5(50)$ \\
\hline VAD & $7(7.5)$ & $6(85.7)$ \\
\hline Endocarditis & $6(6.5)$ & $0(0)$ \\
\hline Aortic aneurysm & $5(5.4)$ & $0(0)$ \\
\hline TAVR & $5(5.4)$ & $1(20)$ \\
\hline Orthotopic heart transplant & $4(4.3)$ & $3(75)$ \\
\hline MVR & $4(4.3)$ & $2(50)$ \\
\hline CABG and MVR & $2(2.2)$ & $0(0)$ \\
\hline CABG and AVR & $2(2.2)$ & $0(0)$ \\
\hline Rheumatic heart disease & $2(2.2)$ & $0(0)$ \\
\hline \multicolumn{3}{|l|}{ Other procedures } \\
\hline IABP preoperative & $11(11.8)$ & $7(63.6)$ \\
\hline VAD same admission & $10(10.8)$ & $8(80)$ \\
\hline IABP intraoperative & $8(8.6)$ & $2(25)$ \\
\hline Catheter assist device & $8(8.6)$ & $4(50)$ \\
\hline ECMO & $5(5.4)$ & $5(100)$ \\
\hline Atrial fibrillation procedure & $5(5.4)$ & $2(40)$ \\
\hline Previous VAD & $4(4.3)$ & $4(100)$ \\
\hline Other noncardiac vascular surgery & $4(4.3)$ & $2(50)$ \\
\hline Other noncardiac thoracic surgery & $3(3.2)$ & $1(33.2)$ \\
\hline IABP postoperative & $2(2.2)$ & $0(0)$ \\
\hline Other noncardiac endarterectomy & $1(1.1)$ & $1(100)$ \\
\hline
\end{tabular}

\section{METHODS}

This is a retrospective chart review using a single academic institution's data submitted to the Society of Thoracic Surgeons (STS) National Database. All patients with operative mortality after cardiac surgery performed between January 1 , 2013, and August 31, 2018, were included.

The STS National Database is a voluntary clinical registry created to facilitate a national quality improvement and safety initiative for cardiothoracic surgery. It has been used as a model for many other specialty organizations and was one of the first attempts at providing surgeons with nationally benchmarked, risk-adjusted outcomes. The database reports
Table 2. Characteristics of Study Participants* [CONT.]

\begin{tabular}{lcc}
\hline & All & $\begin{array}{c}\text { Palliative Care } \\
\text { Consultation }\end{array}$ \\
\hline $\begin{array}{l}\text { Prior cardiac surgery } \\
\text { Redo cardiac surgery } \\
\text { Status at time of admission }\end{array}$ & $29(31.2)$ & $13(44.8)$ \\
$\quad$ Elective & $35(37.6)$ & $10(28.6)$ \\
Urgent & $27(29)$ & $12(44.4)$ \\
Emergent & $23(24.7)$ & $6(26)$ \\
Emergent salvage & $8(8.6)$ & $1(12.5)$ \\
Discharge location & & \\
Died in hospital & $79(84.9)$ & $23(29)$ \\
Home & $6(6.5)$ & $1(16.7)$ \\
Long-term care facility & $3(3.2)$ & $1(33.3)$ \\
Hospice & $5(5.4)$ & $3(60)$ \\
\hline
\end{tabular}

*Data are $\mathrm{n}(\%)$. MVR indicates mitral valve replacement; TAVR, transcatheter aortic valve replacement.

on 7 major cardiac procedures, and all STS data are subject to internal validation checks. Data managers at each site abstract information from the medical record into a standardized data collection form. In addition to operative mortality, the database reports specialty-specific complications, postoperative length of stay, and 30-day readmission, as well as demographic information and granular specialty-specific covariates [Farjah 2018].

Operative mortality was defined as death within 30 days of surgery or during the same hospital admission. For the purposes of this article, we decided to look at the following demographics and variables: age, sex, race, body surface area, other comorbid conditions at the time of surgery, surgery type, other procedures during same admission, prior cardiac surgeries, status at time of admission, and discharge location reported to the STS database. A detailed chart review was performed on all patients. A patient was considered to have a palliative care consultation only if the palliative care service was documented in the medical record. The time of first palliative care interaction was defined as the date of service associated with the first palliative care documentation note. Patients were excluded if transferred from an outside facility and did not survive long enough to undergo cardiac surgery. This study was reviewed and deemed exempt from institutional review board approval at Oregon Health and Science University.

Basic statistical measures were calculated for each variable. Results were reported as a proportion of the entire operative mortality cohort, and palliative care consultations were reported as a percentage of those individuals among each variable with a palliative care consultation. Given the small number of palliative care consultations, this was the most meaningful and representative way to report the data. 
Table 3. Status at Time of Admission Definitions According to the STS

The patient's cardiac function has been stable in the days or weeks before the operation. The procedure could be deferred without
Elective
increased risk of compromised cardiac outcome.
Prgent
Patient requires emergency operations that will have ongoing, refractory (difficult, complicated, and/or unmanageable) unrelenting
Emergent
cardiac compromise, with or without hemodynamic instability, and is not responsive to any form of therapy except cardiac surgery. An
emergency operation is one in which operative intervention should not be delayed.

\section{RESULTS}

Ninety-six patients were identified. Three patients were excluded who were transferred from an outside hospital for evaluation and placement of an intra-aortic balloon pump (IABP) but did not undergo definitive cardiac surgery. Thus, our total study population was 93 patients with operative mortality.

The mean age at the time of surgery was 64 years (range 24 to 88 ), and $67 \%$ of patients were male. The most common comorbidities included hypertension (44.8\%), diabetes (36.5\%), and dyslipidemia (35.4\%) (Table 1$)$.

The most common surgeries were aortic dissection $(19.3 \%)$, coronary artery bypass graft (CABG) $(16.1 \%)$, and aortic valve replacement (AVR) (10.8\%) (Table 2). Twentynine patients $(31.2 \%)$ had prior cardiac surgery. Surgeries were classified as being elective, urgent, emergent, or emergent salvage $(37.5 \%, 28.1 \%, 24 \%$, and $8.3 \%$, respectively) (STS definitions provided in Table 3). Discharge locations included in-hospital death, home, hospice, and long-term care facility $(85.4 \%, 6.3 \%, 5.2 \%$, and $3.1 \%$, respectively).

Twenty-eight patients $(30.1 \%)$ had a palliative care consultation (3 preoperative, 20 postoperative, and 5 both). The median time from first palliative care involvement to death was 6 days (range 0 to 463). The surgery types with the greatest percentage of palliative care involvement were ventricular assist device (VAD) (85.7\%), heart transplantation $(75 \%)$, and AVR (50\%). Other procedures performed during the same admission with the greatest percentage of palliative care involvement included preoperative VAD placement $(100 \%)$, postoperative extracorporeal membrane oxygenation (ECMO) (100\%), and postoperative VAD placement $(80 \%)$.

Urgent surgeries had the greatest percentage of patients with a palliative care consultation $(44.4 \% ; 66.7 \%$ of consultations occurring postoperatively). Sixty percent of patients discharged to hospice, $33.3 \%$ of patients discharged to a long-term care facility, $30.5 \%$ of patients who died in the hospital, and $16.7 \%$ of patients discharged home had palliative care involvement. procedures performed on this cohort are not included in the STS risk calculation. This study was intended to capture the involvement of palliative care in patients with high mortality risk, by focusing on those who died after cardiac surgery. Not all patients who suffer cardiac mortality are considered at high operative risk, and thus it is likely that many high-risk patients who survived cardiac surgery were not included in this study.

It is striking that only $30 \%$ of patients with operative mortality had a palliative care consultation, with just 8 consultations $(28.6 \%)$ occurring preoperatively. In this study, palliative care was often involved late in the disease trajectory or when death was imminent, with a median time from consultation to death of only 6 days.

Nearly $40 \%$ of patients with operative mortality in this cohort were classified as elective, yet only 1 patient (2.8\%) had a preoperative palliative care consultation. Based on the definition used by the STS, those patients with an elective status should inherently have more time, and thus opportunities, for palliative care involvement. Our findings suggest that patient mortality risk for elective surgery is being underestimated, or the indications for palliative care consultation in high-risk patients is going unrecognized.

It is assumed that most patients who die after cardiac surgery would have benefited from a palliative care consultation. Similarly, high-risk patients with a favorable survival outcome may also benefit from palliative involvement but were not captured in this study. It was beyond the scope of this study to evaluate the effect of palliative care involvement on cardiac surgery patients.

We recognize that this cohort represents a unique population in that it includes only those with operative mortality. Subsequent investigations should focus on the incidence of palliative care consultation for all high-risk patients with varying surgical outcomes, as well as high-risk patients who were considered for but did not undergo cardiac surgery. Future studies should also investigate the impact of palliative care consultation on patient satisfaction and decision-making. Such knowledge may facilitate earlier palliative care involvement, with the goal of improved quality of life for cardiac patients.

\section{DISCUSSION}

Cardiac patients are routinely evaluated for mortality risk preoperatively using the STS Predicted Risk of Mortality (STS-PROM) calculator. Unfortunately, most cardiac

\section{REFERENCES}

Diop MS, Rudolph JL, Zimmerman KM, Richter MA, Skarf LM. Palliative care interventions for patients with heart failure: A systematic review and meta-analysis. J Palliat Med 2017;20:84-92. 
Farjah F, Kaji AH, Chu D. Practical guide to surgical data sets: Society of Thoracic Surgeons (STS) National Database. JAMA Surg 2018;153:955-956.

Lilley EJ, Cooper Z, Schwarze ML, Mosenthal AC. Palliative care in surgery: Defining the research priorities. Ann Surg 2018;267:66-72.

Warraich HJ, Allen LA, Mukamal KJ, Ship A, Kociol RD. Accuracy of physician prognosis in heart failure and lung cancer: Comparison between physician estimates and model predicted survival. Palliat Med
2016;30:684-689.

Warraich HJH, Hernanedez AF, Allen, LA. How medicine has changed the end of life for patients with cardiovascular disease. J Am Coll Cardiol 2017;70:1276-1289.

Warraich HJ, Wolf SP, Mentz RJ, Rogers JG, Samsa G, Kamal AH. Characteristics and trends among patients with cardiovascular disease referred to palliative care. JAMA Netw Open 2019;2:e192375. 